\title{
ROSETTA-informed design of structurally stabilized cyclic anti-amyloid peptides
}

Chandler Est, Parth Mangrolia and Regina Murphy Department of Chemical and Biological Engineering University of Wisconsin Madison, WI, 53706

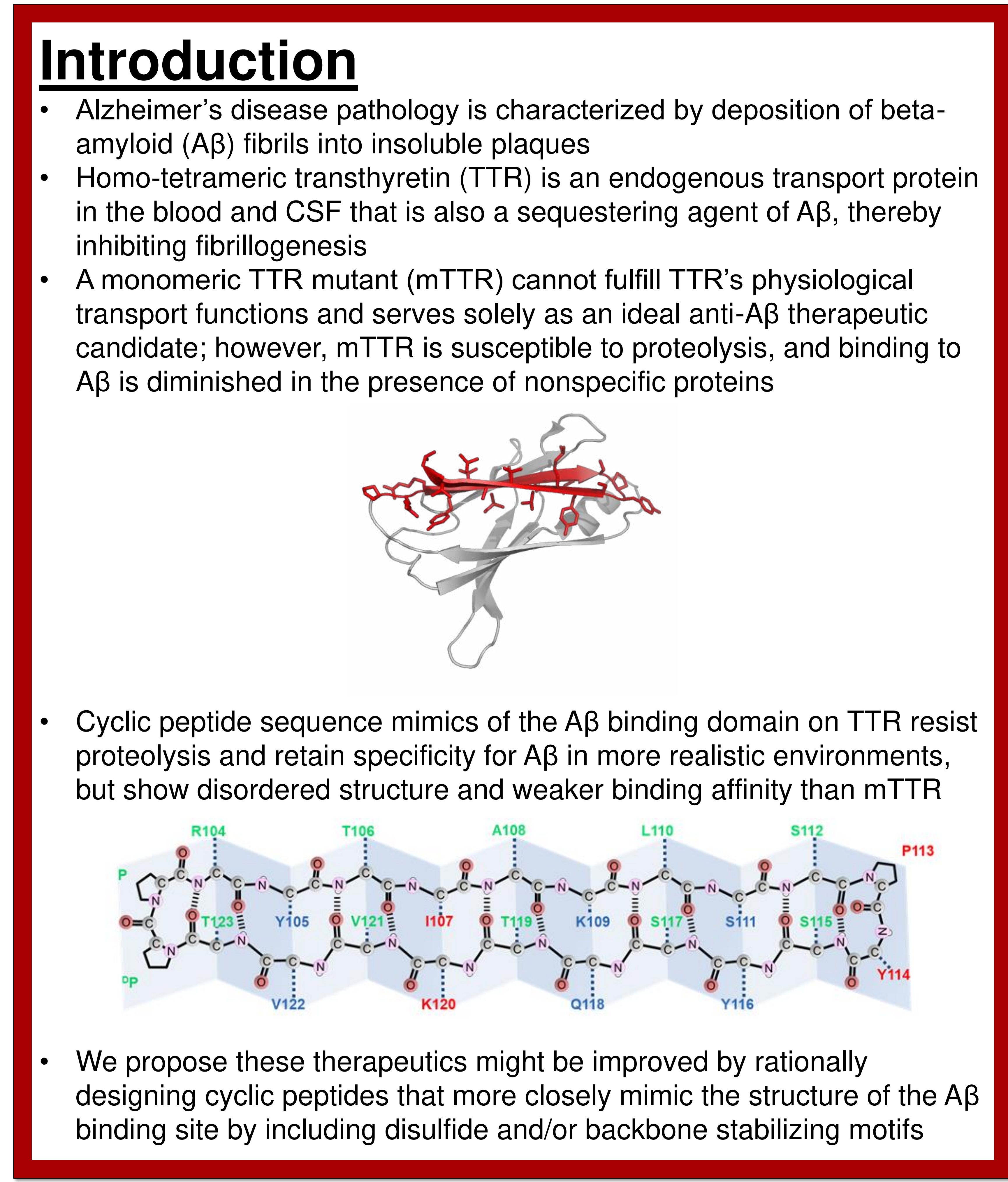

\section{Methodology}

In silico, rational design of stabilized cyclic peptide structures using the Simple Cyclic Peptide Application (SCPA) of the ROSETTA software suite

Validation of SCPA predicted secondary structure by circular dichroic

- Characterization of anti-amyloid efficacy by Thioflavin T (ThT) assay

\section{Acknowledgements \& References - Biotechnogy Training Program, funded by NIH 5 T32 GM008349 NIH R01AG033493 \\ Darrell McCaslin for help in CD experimental design Est, C. B., et al. (2019). Protein Eng Des Sel 32(2): 47-57
Est}

\section{ROSETTA SCPA Simulation Results}

cG8 (black - original cyclic peptide); $c G 8_{s s}$ (blue - putative disulfide and cyclic peptide); $c G \delta_{d P r o p r o}$ (green - further cyclically constrained peptide).

\author{
A)
}

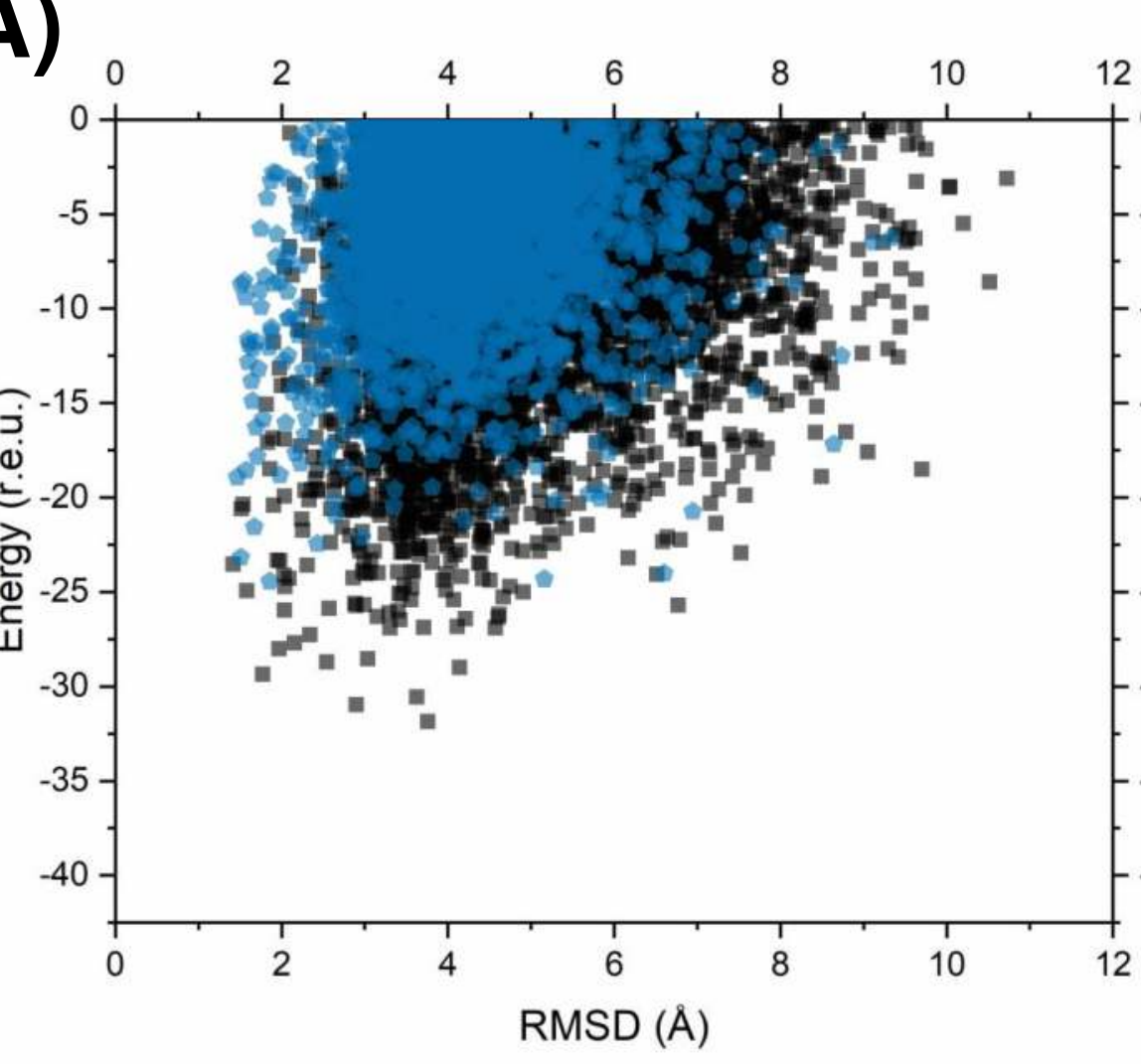

B)

C)

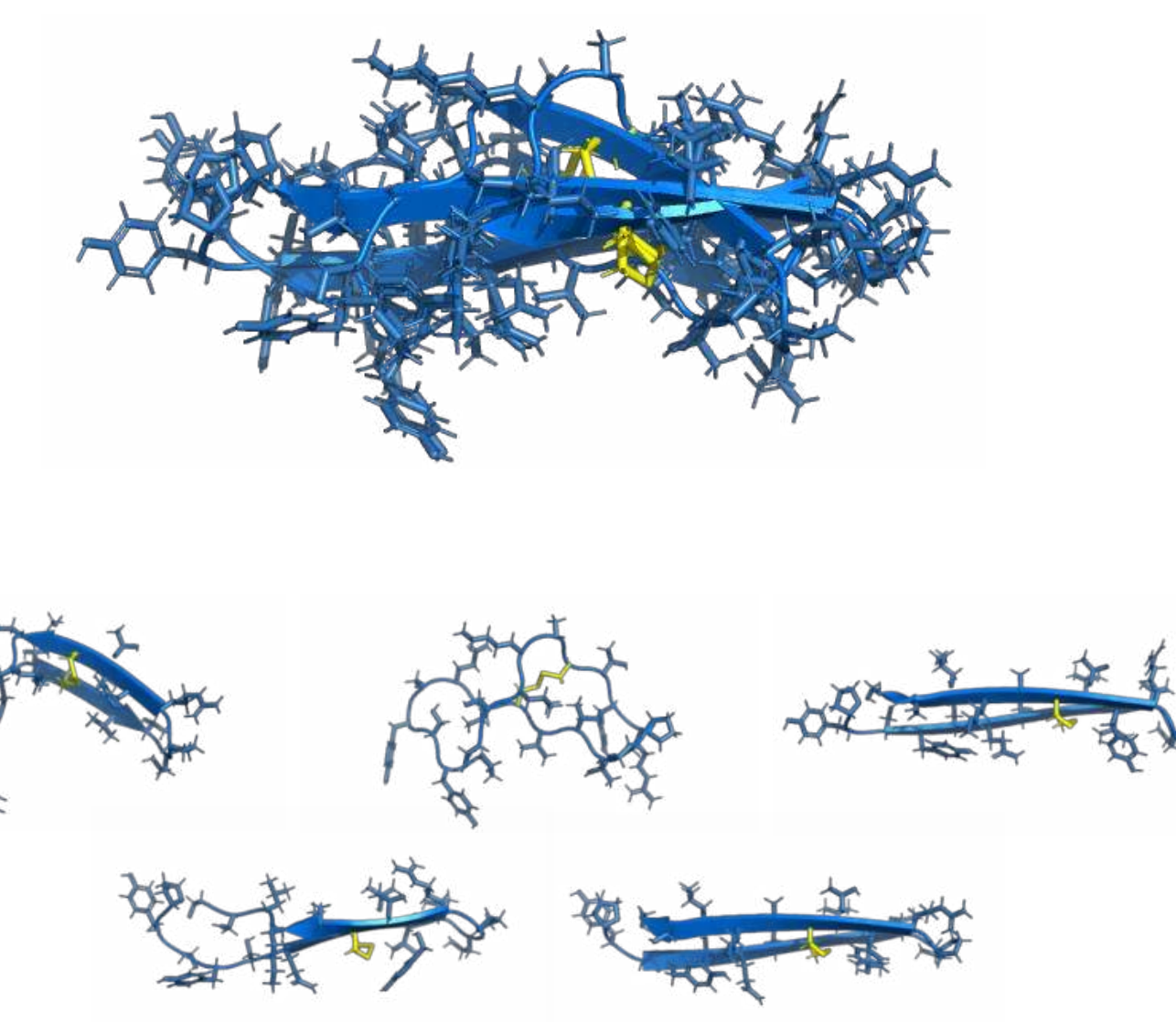

A)

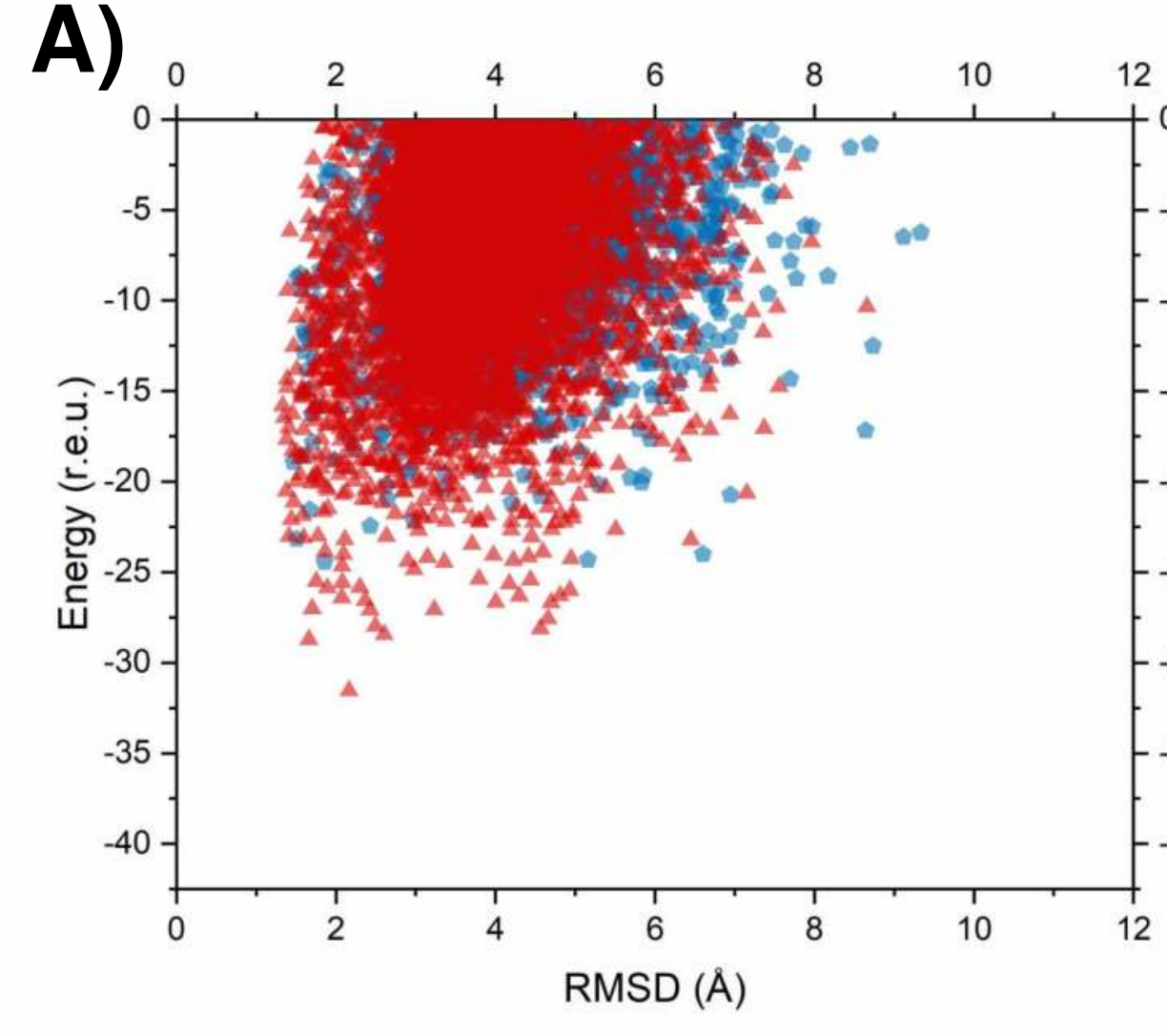

B)
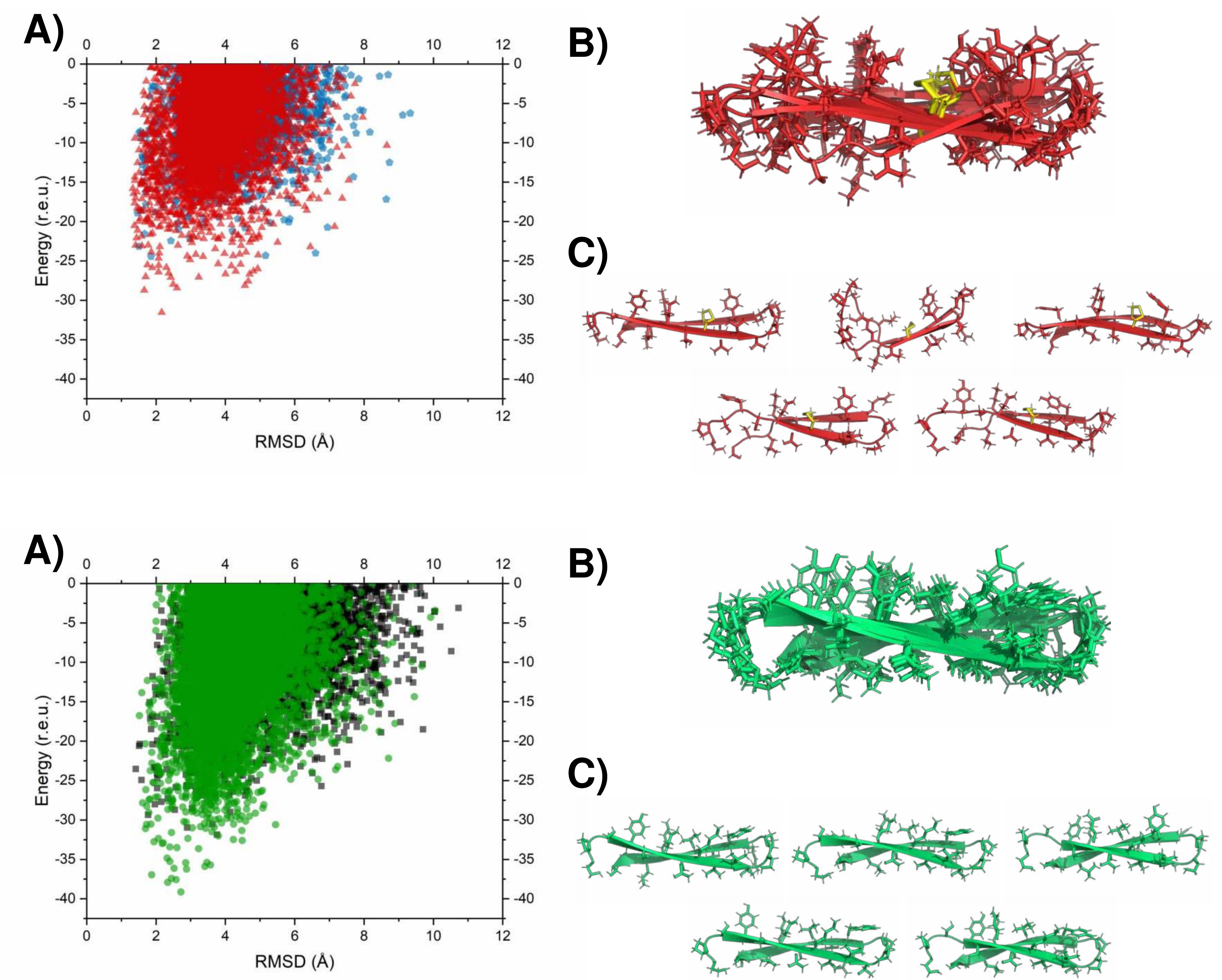

B)

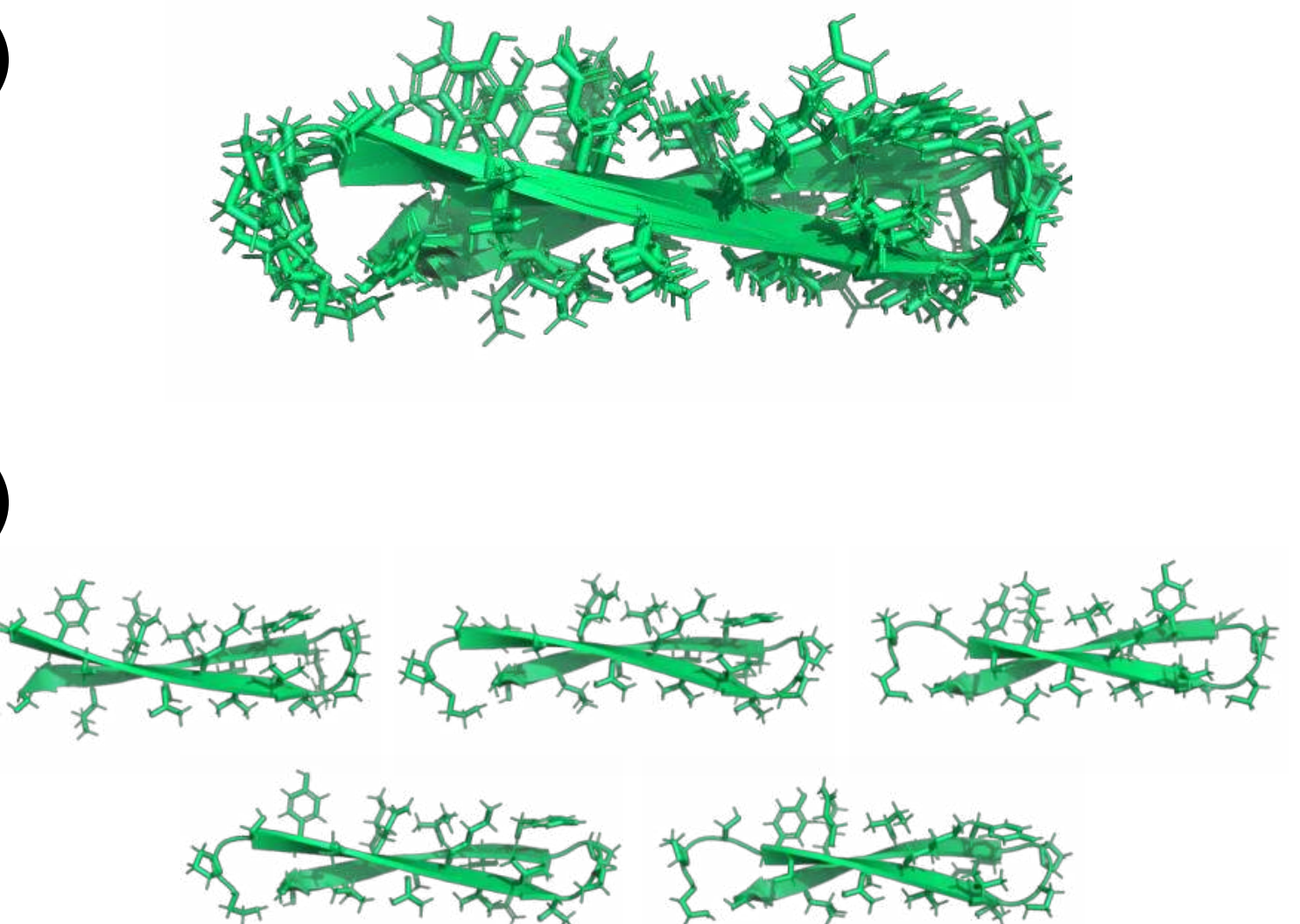

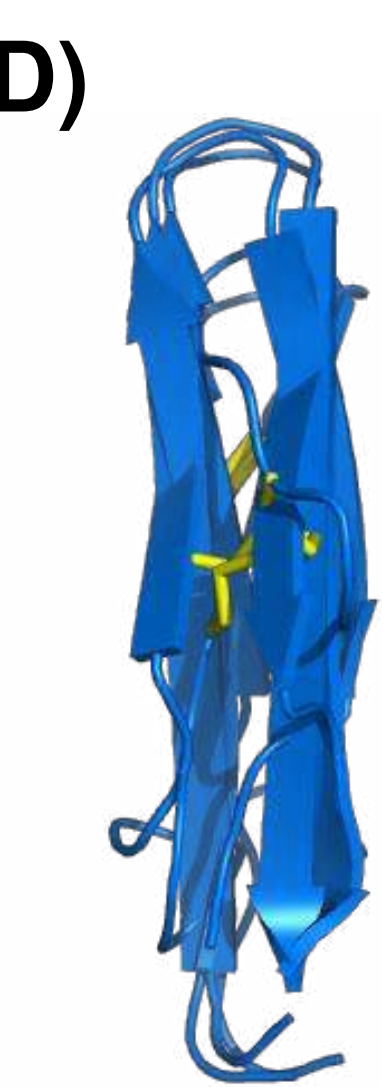

D)

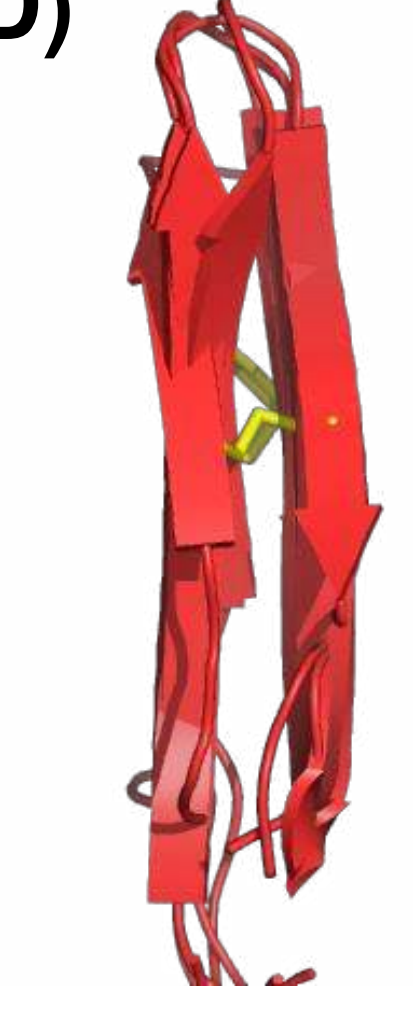

D)

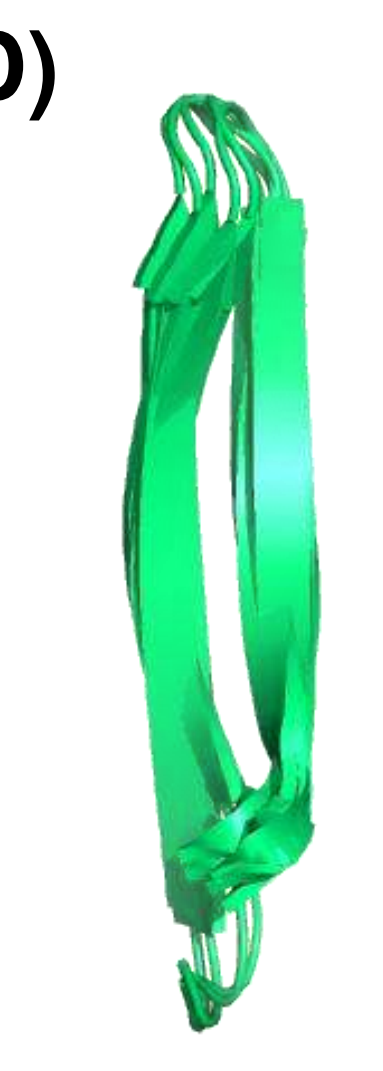

A) Comparison of energy values and atomic RMSD for 10,000 independent completed closures. B) Cartoon overlay showing residue side chains of the five lowes energy structures from the full simulation. C) Cartoons showing residue side chains of each of the five lowest energy structures individually. D) Cartoon overlay of the five west energy structures with residue side chains hidden.

\section{Experimental Validation}

cG8 (black - original cyclic peptide); $c G 8_{s s}$ (blue - putative disulfide and

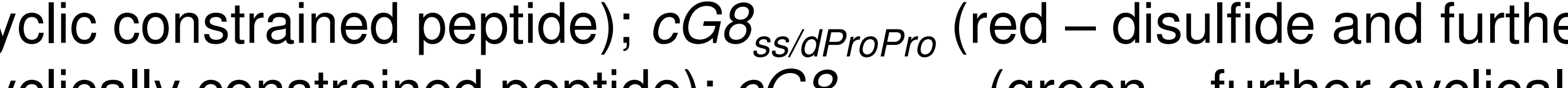
constrained peptide).

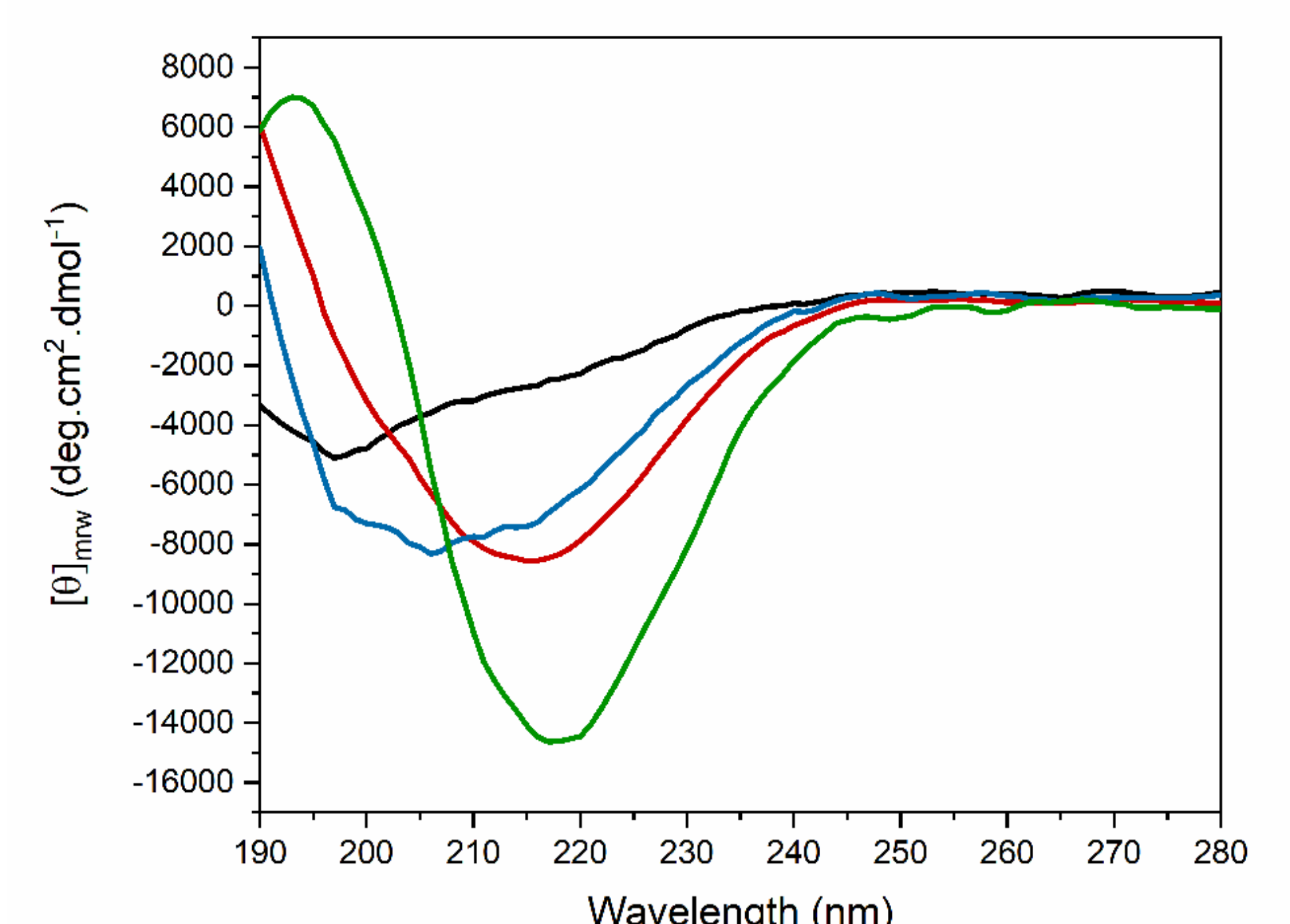

CD spectra at a peptide concentration of $45 \mu \mathrm{M}$ in $10 \mathrm{mM}$ sodium phosphate, $140 \mathrm{mM} \mathrm{NaF}$, pH 7.4 buffer.

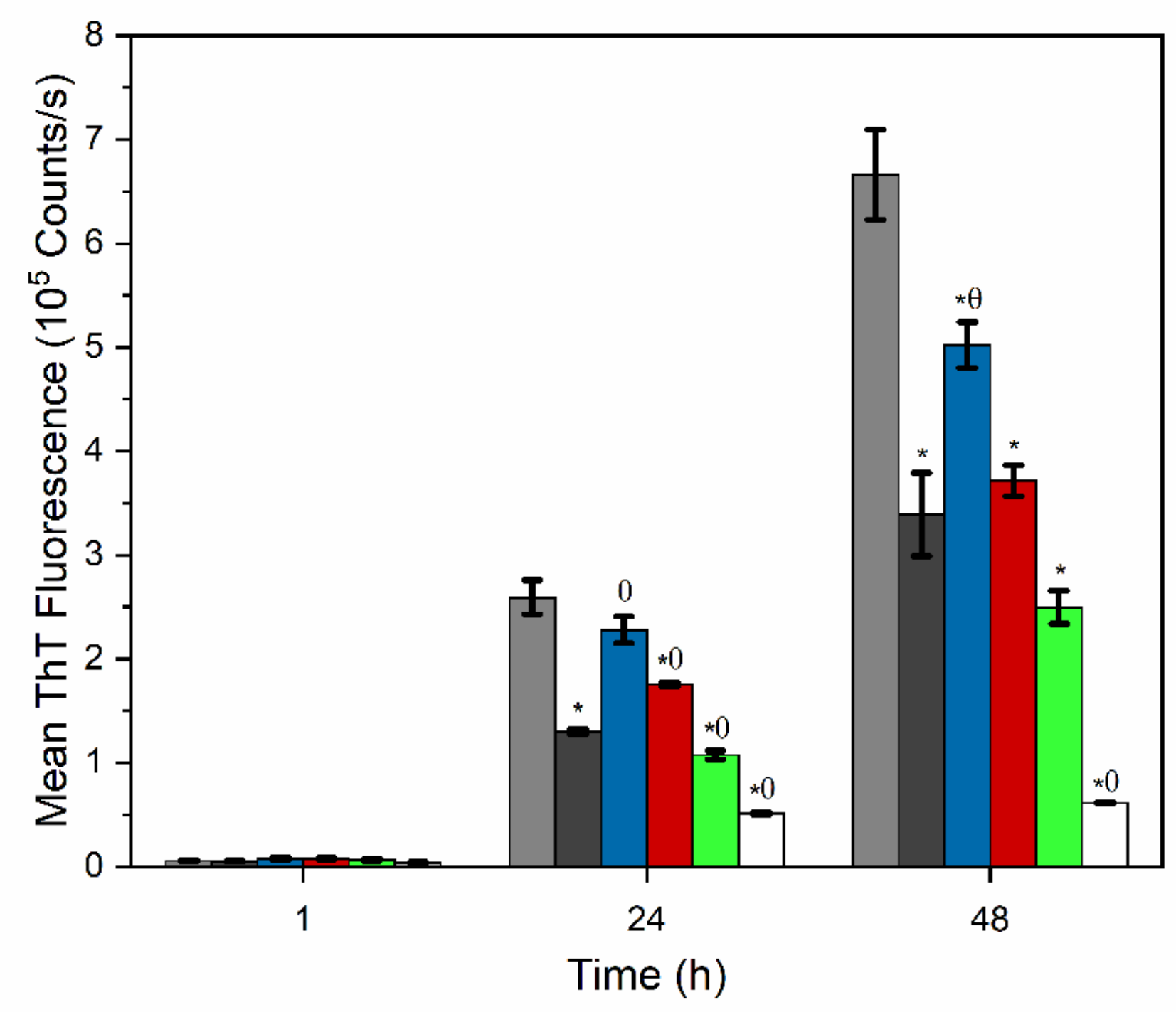

A fibril formation, as measured by ThT fluorescence intensity, alone or in the presence of designed therapeutics at a 20:1 (A $\beta$.therapeutic) molar ratio, at 1,24 or $48 \mathrm{~h}$. Data were taken at $37^{\circ} \mathrm{C}$. All data are background subtracted, and error bars indicate SEM. $\left(^{*}\right)$ indicates different vs. $A \beta$ alone $(P<0.05) ;(\theta)$ indicates different vs. cG8 $(P<0.05)$. All peptides were ThTnegative in the absence of $A \beta$. (Gray, no therapeutic; white, mTTR)

\section{Conclusions}

Disulfide constraint of the peptide backbone has minimal effect on stability or anti-amyloid efficacy

Incorpor
efficacy

Regardless, cyclization, $\beta$-sheet structure and conformational homogeneity are preferable design features of anti-amyloid peptides - Lack of enhanced anti-amyloid efficacy may suggest $\mathrm{CG}_{\mathrm{dPropro}}$ does not adopt the proper conformation for maximal $\mathrm{A} \beta$ binding - Recent solution phase NMR spectra (right, adapted from Oroz et al) indicate the structure of MTTR in solution may not be as rigid as previously believed, suggesting next generation peptides may need design against a different backbone structure template

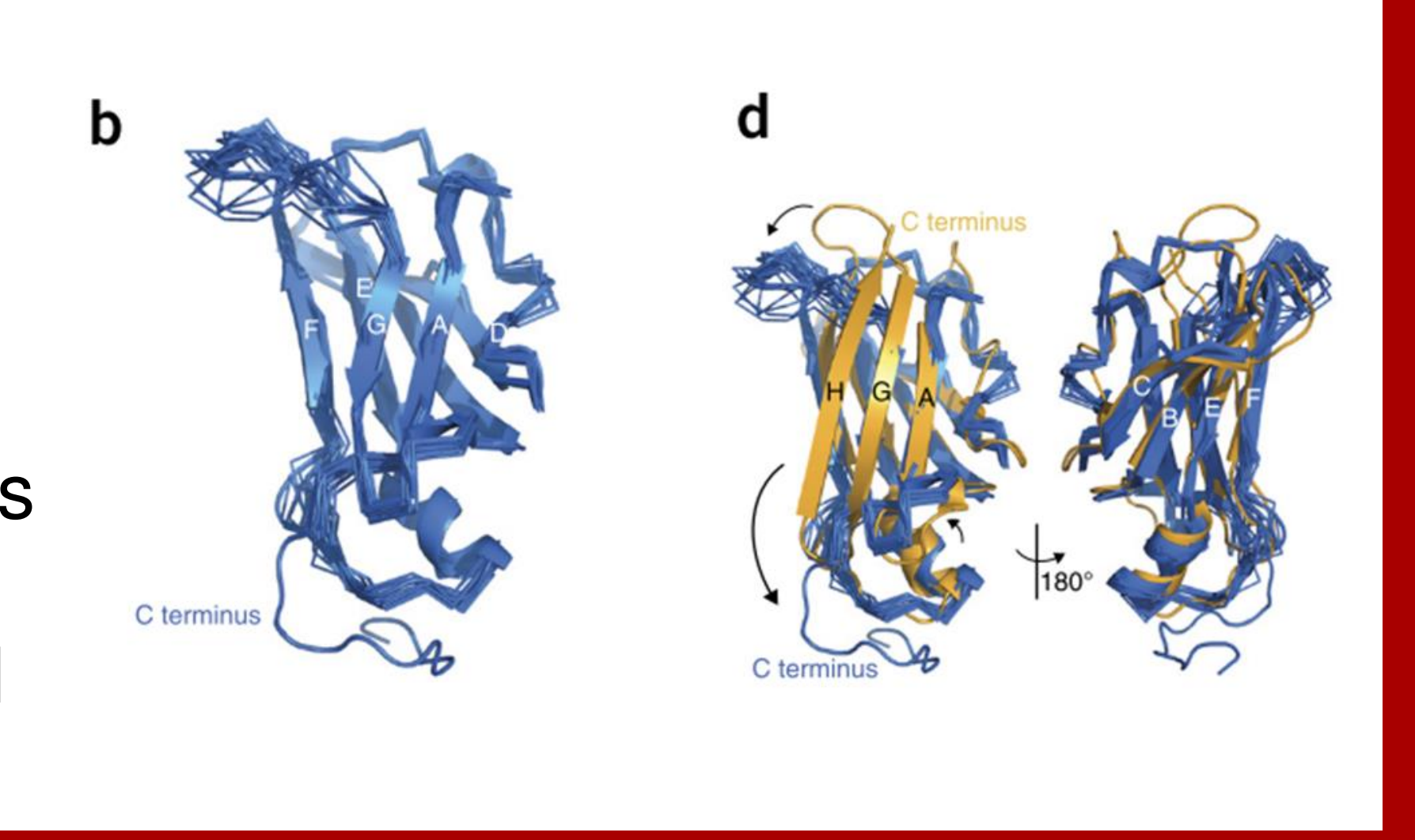

